\title{
A Functional Mathematical Model to Simulate the Single-Breath Nitrogen Washout
}

\author{
Paolo Barbini ${ }^{1, *}$, Chiara Brighenti ${ }^{2}$ and Gianni Gnudi ${ }^{3}$ \\ ${ }^{1}$ Dipartimento di Biotecnologie Mediche, Università di Siena, Viale Mario Bracci 12, 53100 Siena, Italy \\ ${ }^{2}$ Dipartimento di Ingegneria Civile, Chimica, Ambientale e dei Materiali, Università di Bologna, Viale Risorgimento 2, \\ 40136 Bologna, Italy \\ ${ }^{3}$ Dipartimento di Ingegneria dell'Energia Elettrica e dell'Informazione “Guglielmo Marconi”, Università di Bologna, \\ Via Venezia 52, 47521 Cesena, Italy
}

\begin{abstract}
A nonlinear dynamic model is proposed to reproduce and interpret the influence of pulmonary inhomogeneities on the single-breath nitrogen washout (SBNW) curve. The model is characterized by two parallel zones. In each zone, the upper airways are described by a Rohrer resistor. Intermediate airways are represented as a collapsible segment, the volume of which depends on transmural pressure. Smaller airways are described by a resistance which increases when transpulmonary pressure decreases. The respiratory region is modeled as a Voigt element. Three different conditions were simulated: a reference case, characterized by airway-parameter values for normal conditions, and two pathological states corresponding to different levels of disease. In the reference case, a straight line was a good approximation of SBNW phase III and the last point of departure of the nitrogen trace from this line unambiguously identified the onset of phase IV. The slope of phase III rose with disease severity (from a 1.1\% increase in nitrogen concentration per $1000 \mathrm{ml}$ of expired volume in the reference case to $3.6 \%$ and $7.7 \%$ in the pathological cases) and the distinction between phases III and IV became less evident. The results obtained indicate that the slope of phase III depends primarily on nitrogenconcentration differences between lung zones, as determined by different mechanical properties of the respiratory airways. In spite of the simplified representation of the lungs, the similarity of the simulation results to actual data suggests that the proposed model describes important physiological mechanisms underlying changes observed during SBNW in normal and pathological patients.
\end{abstract}

Keywords: Airway mechanical properties, breathing mechanics, lung inhomogeneity, mathematical model, single breath nitrogen washout.

\section{INTRODUCTION}

The single-breath nitrogen washout test provides three useful pieces of information: (a) an estimate of anatomical dead space; (b) an assessment of the distribution of ventilation, and (c) a measure of closing volume [1]. In this test the patient takes a single vital capacity inspiration of pure oxygen and then exhales slowly to residual volume (RV). The exhaled volume and the nitrogen concentration in that volume are measured to obtain the nitrogen-washout curve. The shape of the curve is generally characterized by four phases [2]. At the beginning of expiration (phase I), lung volume changes but the nitrogen concentration in the exhaled volume is zero, as nitrogen-free gas (pure oxygen) leaks out of the anatomical dead space. Then there is a sudden increase in nitrogen concentration making the nitrogen washout curve Sshaped (phase II). This phase represents the transition between emptying of the dead space and the arrival of mixed alveolar gas. During midexpiration, when mixed alveolar

*Address correspondence to this author at the Department of Medical Biotechnology, University of Siena, Viale Mario Bracci 12, 53100 Siena, Italy; Tel/Fax:+39 0577 585797; E-mail: paolo.barbini@unisi.it gas is emptied from all parts of the lungs, the nitrogen washout curve is relatively straight (phase III). The expired gas of the initial part of phase III usually has a lower nitrogen concentration than that of the latter part, so that a gas concentration change is observed during this phase. Finally, an abrupt rise in nitrogen concentration is observed at the end of expiration (phase IV) due to a drastic change in rates of emptying of different parts of the lungs. During phase III, the average slope of the curve for normal lungs does not exceed a $1.5 \%$ increase in nitrogen concentration per $500 \mathrm{ml}$ of expired volume for an interval of expired volume between 750 and 1250 $\mathrm{ml}$ below total lung capacity [2]. Increases in phase III slope are generally regarded as a marker of small airway alterations. This slope is influenced by a variety of factors [3], but the quantitative effect of the different mechanisms is not clear. A key mechanism seems related to inhomogeneities in the conducting airways at branch points, generating concentration differences between parallel lung units, which fill and empty in sequence. Another more peripheral mechanism may occur in the intra-acinar airways, where asymmetry of acinar structure generates intra-acinar concentration differences. 
Approaches based on lung models have been widely used to reproduce the effects of lung inhomogeneities on the single breath washout test since Pardaens et al, [4] simulated asymmetries in the lung-airways system using a model with two parallel branches having the dimensions of Weibel's [5] fifth bronchial generation and elastic properties representative of bronchioles. In particular, various studies have been carried out to investigate the generation of phase III assuming asymmetry at acinar level [6-9]. Dutrieue et al, [9] analyzed the intra-acinar contribution to the alveolar plateau for gases of differing diffusivities, using a multi-branch-point model of the human acinus that took diffusive and convective gas transport into account. They proved that phase III slope increases were greatest when asymmetry and variability of asymmetry increased in airways of the respiratory region characterized by different gas diffusion fronts. The study is interesting because it points out the sensitivity of phase III slope to the diffusive mechanism in asymmetric airways. However it does not consider the role of inhomogeneities in conducting airways that may greatly influence the phase III time course.

Kaneko [10] proposed a model characterized by an upright lung with 12 horizontally sliced regions to simulate simultaneous helium and nitrogen single-breath washout. Although the model aims to account for the effects of inhomogeneous lung elasticity and unequal critical airway closing pressure, it does not completely consider mechanisms due to inhomogeneities occurring at branch points in the conducting airways and is limited to analysis of the quasi-static condition. A much more anatomically accurate three-dimensional model was used to directly analyze the influence of conducting airway asymmetry on the slope of the alveolar plateau in phase III of the gas washout curve normalized by the average expired concentration of resident gas [11]. However, despite its complexity, the model considers each branch of the bronchial tree as a cylinder of constant diameter and therefore does not account for dynamic airway modifications occurring during inspiration and expiration. An accurate morphometric description of the tracheobronchial tree would actually require a vast number of model equations and parameters which would may make it very difficult to consider fundamental phenomena influencing dynamic system behavior. Approaches based on functional models may overcome such problems, providing a realistic, effective and much simpler description of the system.

Arieli et al [12] proposed a functional three-compartment viscoelastic model of the lungs, intended to consider the effect of heterogeneity of pressure-volume properties of lung units and the effect of heart beat on expired nitrogen, but the model ignores the characteristics of the intermediate airways which may strongly influence expiratory pattern [13]. Hook et al [14] compared a variety of elementary functional models for simulating single-breath washout of insoluble gases. Although these models use a very simple description of the bronchial tree, the results indicate that the slope of the alveolar plateau is closely related to models featuring unequal distribution of diffusive conditions and/or unequal specific ventilation of parallel compartments. The basic role played by structural and functional lung inhomogeneities during phase III was confirmed by a paper in which single-breath nitrogen washout curves were simulated using a lung deformation model, in which the lung was regarded as a porous elastic body similar to a sponge [15]. Although the model seems to reproduce the effects of gravity on lung deformation, it fails to mimic phase III slope because it does not account for lung inhomogeneities.

In the present study, we introduce a nonlinear dynamic functional model of breathing mechanics to investigate the manner in which inhomogeneities of the bronchial tree may influence phase III slope. The proposed model, based on previous work $[13,16,17]$, reproduces respiratory mechanics during spontaneous breathing both in tidal breathing and the single-breath nitrogen washout test. It incorporates two parallel zones and accounts for different properties of upper, intermediate and smaller airways, thus taking series and parallel inhomogeneities of the bronchial tree into consideration. Physiological and pathological conditions are simulated to reproduce different single-breath nitrogen washout curves and the role of lung inhomogeneities in determining different shapes is discussed. The simulation results obtained by this model provide useful information for pathophysiological interpretation of the results of single-breath nitrogen washout tests.

\section{METHODS}

In the model, parallel heterogeneity is considered by simply dividing the pulmonary system, including the acini, into two parallel zones, hereafter referred to as Z1 and Z2, characterized by different mechanical properties. Identical values of pleural pressure $\left(P_{p l}(t)\right)$ are assumed throughout the lungs.

The model structure might be easily modified to simulate more complex parallel inhomogeneities, by including more than two parallel zones.

\subsection{Tracheobronchial Tree Model}

For each zone, the tracheobronchial tree is divided into conductive and respiratory regions that include the first 16 generations and the last 7 generations, respectively, of the Weibel representation [5]. The conductive region is further divided into three segments in series, characterized by different mechanical properties:

1. upper airways, i.e. airways between mouth and lobar bronchi (generations 0-4);

2. intermediate airways, which in the present model include the airways between segmental bronchi and small bronchi (generations 5-11) and the first generations (12-14) of terminal bronchioles;

3. smaller airways, i.e. the last conducting airways (generations 15-16).

Since the model equations are the same for each zone, we give the relationships in a generic zone $\mathrm{Zi}$, where $\mathrm{i}=1,2$. All the zones originate from the mouth, therefore total mouth flow rate $(\dot{v})$ is given by the sum of flow rates $\left(\dot{v}_{Z i}\right)$ leaving $\mathrm{Z} 1$ and $\mathrm{Z} 2$.

Upper airways are described as in the Barbini model [13] using a nonlinear Rohrer resistor [18], $R_{u Z i}$ (see Appendix). 
The intermediate airways are represented as a collapsible segment of constant length [13], the volume $\left(v_{c Z i}\right)$ of which varies with transmural pressure $\left(p_{t m_{Z i}}\right)$ according to a sigmoidal elastic curve based on that proposed by Lambert [19] (see Appendix). Elastic and viscous properties of this segment are modeled by non linear elastance $\left(E_{a w Z i}\right)$ and non linear resistance $\left(R_{i Z i}\right)$ given by the equations:

$E_{a w Z i}=\frac{\mathrm{d} p t m_{Z i}}{\mathrm{~d} v_{c Z i}}$

$R_{i Z i}=\frac{k_{3 Z i}}{v_{c Z i}^{2}}$

where $k_{3 Z i}$ is a constant.

Smaller airways are represented by a nonlinear resistance $\left(R_{s Z i}\right)$ which increases when transpulmonary pressure $\left(p_{E I Z i}\right.$, i.e. difference between alveolar pressure and pleural pressure) and consequently alveolar volume decrease. If $p_{E I Z i}$ falls below a set threshold $\left(p_{\mathrm{tszi}}\right)$, the smaller airways are assumed to collapse (see Appendix).

Finally, the respiratory region was modeled as a parallel combination of a spring and dashpot. According to previous findings $[13,16]$, its resistance $\left(R_{l Z i}\right)$ increases as alveolar volume $\left(v_{I Z i}\right)$ decreases, as follows

$R_{I Z i}=\frac{k_{4 Z i}}{v_{I Z i}}$

where $k_{4 Z i}$ is a positive constant.

The elastance of the alveolar space $\left(E_{I Z i}\right)$ is obtained by differentiating $p_{E I Z i}$ with respect to $v_{I Z i}$, where the relationship between $v_{I Z i}$ and $p_{E I Z i}$ is represented by an exponential curve based on the Glaister equation [20] (see Appendix).
The differential equations describing respiratory region dynamics are:

$\frac{\mathrm{d} p t m_{Z i}}{\mathrm{~d} t}=-E_{a w Z i}\left(\dot{v}_{Z i}-\dot{v}_{I Z i}\right) \quad i=1,2$

$\frac{\mathrm{d} p_{E I Z i}}{\mathrm{~d} t}=-E_{I Z i} \dot{V}_{I Z i} \quad i=1,2$

The initial values of $p t m_{Z i}$ and $p_{E I Z i}(i=1,2)$ were assumed equal to $-P_{p l \text { TLC }}$, where $P_{p l \text { TLC }}$ is pleural pressure at TLC.

Flow coming out of the $i$-th zone is

$\dot{v}_{Z i}=\frac{P_{p l}+p t m_{Z i}}{R_{u Z i}+R_{i Z i}}$

while flow coming out of the alveolar space is

$\dot{v}_{I Z i}=\frac{p_{E I Z i}-p t m_{Z i}}{R_{\mathrm{sZi}}+R_{I Z i}}$ for $p_{E I Z i} \geq p_{\mathrm{ts} Z i}$ or $P_{p l} \leq-p_{\mathrm{ts} Z i}$

$\dot{v}_{I Z i}=0$ elsewhere.

The electrical analog of the above-described nonlinear lumped model of the human lung is shown in Fig. (1).

Equations describing how pleural pressure varies during tidal respiration and during the single breath nitrogen washout test are reported in Appendix.

\subsection{Nitrogen Concentration During the Single-breath Washout}

In this model, it is assumed that the conductive region contains pure oxygen at the end of the vital-capacity inspiration of pure oxygen, while both oxygen and nitrogen are present in the respiratory region. The quantity of nitrogen con-

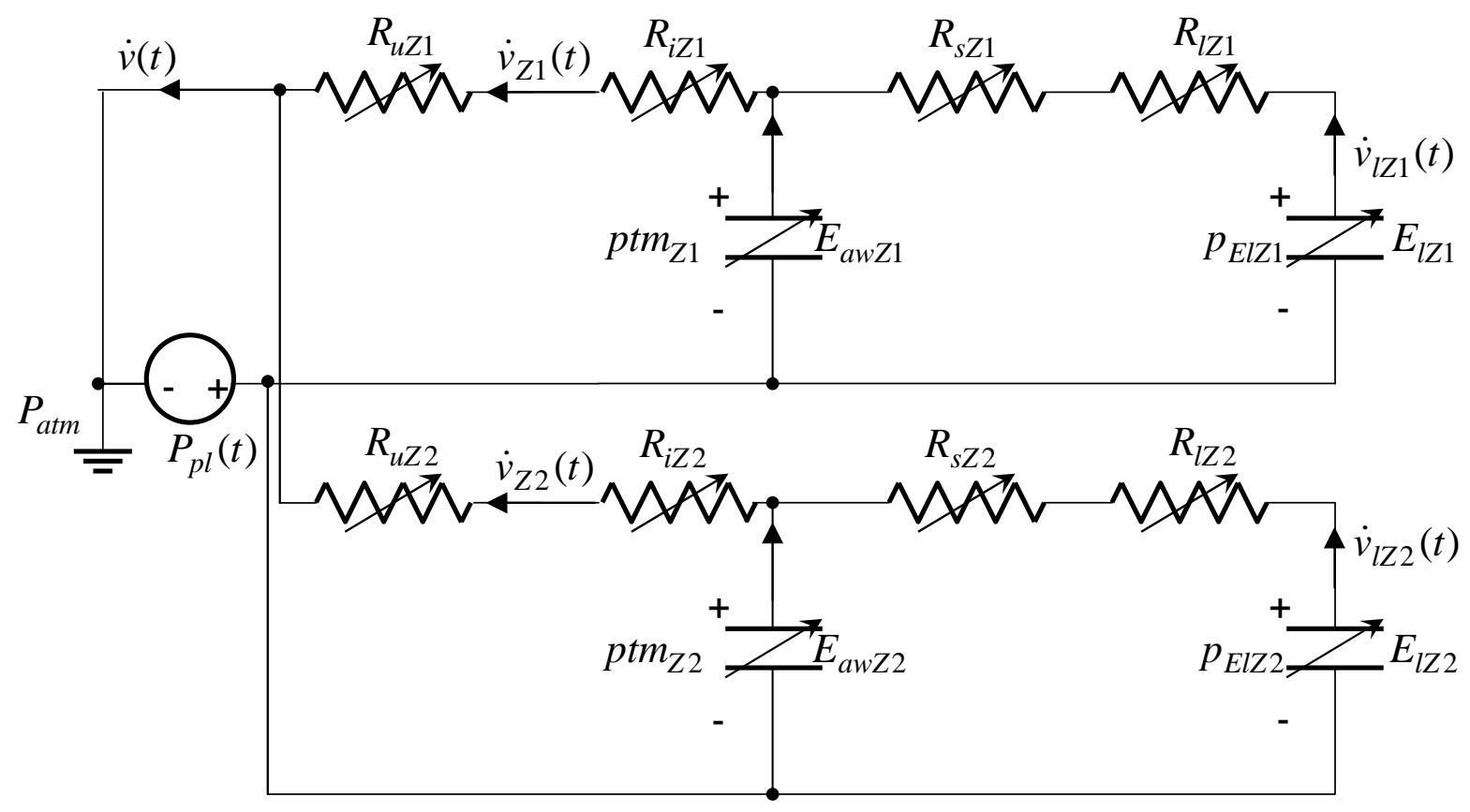

Fig. (1). Electrical analog of the nonlinear lumped model of the human lung. Parallel heterogeneities are taken into account by dividing the pulmonary system into two parallel zones, while series heterogeneities are described by different series segments of the tracheobronchial tree. 
tained in the respiratory region of each zone, $\mathrm{Zi}$, is equal to that contained in $\mathrm{Zi}$ before the vital-capacity inspiration, i.e. equal to the nitrogen fractional concentration in air (assumed equal to 0.8 ), multiplied by $\mathrm{RV}_{Z i}$ (the volume of $\mathrm{Zi}$ at $\mathrm{RV}$ ). If we consider that the air volume of the global respiratory region at TLC is very near to total lung capacity, the nitrogen fractional concentration in the respiratory region of each zone at the end of the vital-capacity inspiration of pure oxygen can be calculated as

$$
F_{\mathrm{N} 2 I Z i}=0.8 \cdot \frac{\mathrm{RV}_{Z i}}{\mathrm{TLC}_{Z i}}
$$

where $\mathrm{TLC}_{Z i}$ is the volume of $\mathrm{Zi}$ at TLC.

To investigate the manner in which inhomogeneities in the bronchial tree may influence emptying of mixed alveolar gas from all parts of the lungs, diffusive and convective gas mixing was assumed to be approximately complete within the respiratory region of each separate zone at the beginning of phase III. Hence, the fractional concentration of nitrogen in the respiratory region of the $i$-th zone $\left(F_{\mathrm{N} 2 Z Z i}\right)$ was assumed

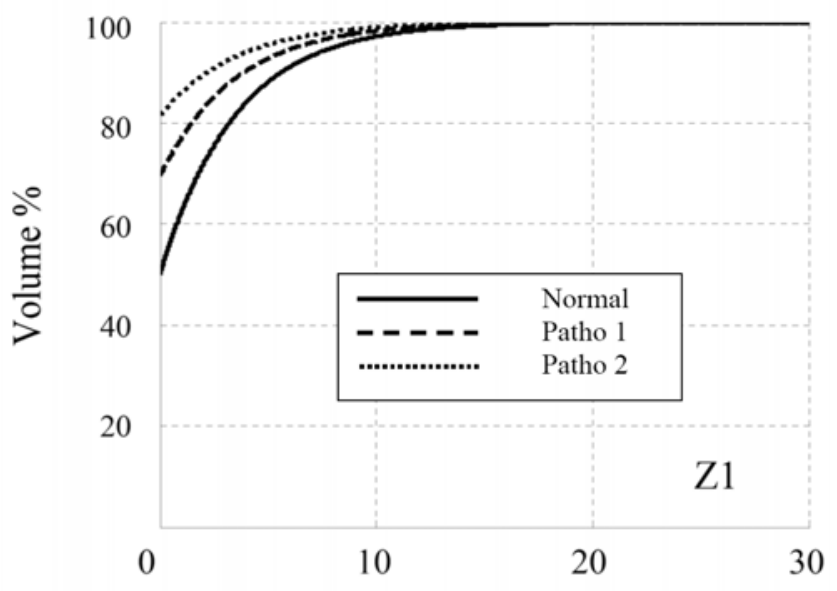

Transpulmonary pressure $\left(\mathrm{cmH}_{2} \mathrm{O}\right)$

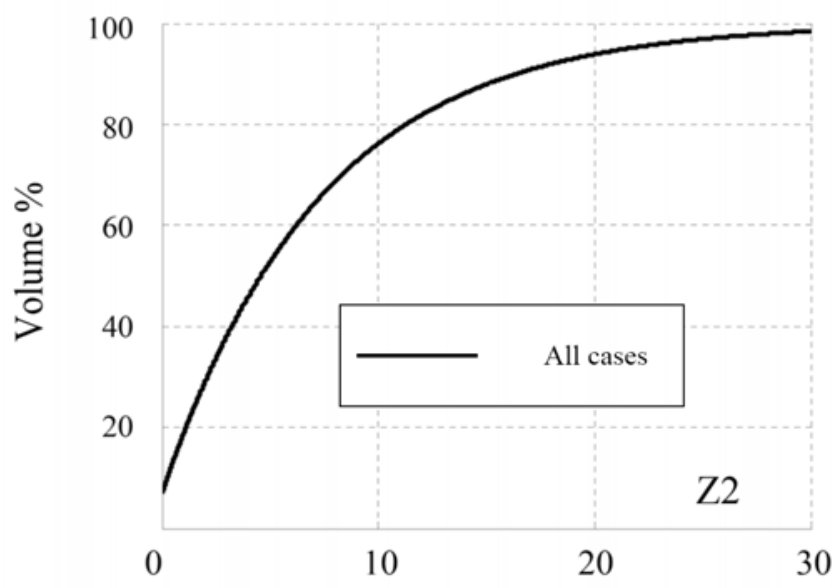

Transpulmonary pressure $\left(\mathrm{cmH}_{2} \mathrm{O}\right)$

Fig. (2). Elastic characteristics of the respiratory region of lung zones $\mathrm{Z} 1$ and $\mathrm{Z} 2$ in normal and pathological cases. The percentage volume of each zone (with respect to its maximum admissible volume) is plotted against transpulmonary pressure. constant during subsequent expiration, and the time course of fractional concentration of nitrogen in expired mixed gas $\left(F_{\mathrm{N} 2}\right)$ was calculated as the flow-weighted mean of concentrations leaving each zone, i.e.

$$
F_{\mathrm{N} 2}=\frac{\sum_{i=1}^{2} \dot{v}_{I Z i} \cdot F_{\mathrm{N} 2 l Z i}}{\sum_{i=1}^{2} \dot{v}_{I Z i}}
$$

Starting from the beginning of phase III, equation (9) shows that the concentration of nitrogen would be constant if its fractional concentration in $\mathrm{Z} 1$ and $\mathrm{Z} 2$ was the same (i.e. in the absence of inhomogeneities), irrespective of the airflows from the two zones. However, when the nitrogen concentrations in the two zones are different, the time course of the resulting concentration in the expired gas also depends on the airflows from the two zones. In fact, the total airflow rate from the respiratory region $\dot{v}_{l}=\dot{v}_{I Z 1}+\dot{v}_{I Z 2}$ can be considered constant, because the total expiratory flow is constant throughout the slow vital-capacity expiration. Thus the slope of the nitrogen concentration curve can be written as

$\frac{d F_{\mathrm{N} 2}}{d v_{l}}=\frac{\frac{d \dot{v}_{l Z 1}}{d t}}{\left(\dot{v}_{l}\right)^{2}}\left(F_{\mathrm{N} 2 l Z 1}-F_{\mathrm{N} 2 l Z 2}\right)$

where $v_{l}=v_{I Z 1}+v_{I Z 2}$.

Equation (10) shows that the slope depends on the concentration difference, the total airflow rate and the time variation of airflow from Z1. Of course, $\frac{d \dot{v}_{I Z 1}}{d t}=-\frac{d \dot{v}_{I Z 2}}{d t}$ because the total expiratory airflow is constant.

\subsection{Simulation Setting}

Three different conditions were simulated by the nonlinear model of Fig. (1). A first case (Normal in Table 1) was defined as reference and was characterized by airwayparameter values for normal conditions of breathing mechanics during spontaneous ventilation (see West [2]). Next, we considered two altered conditions, in an attempt to simulate two levels of disease that may exhibit increased lung inhomogeneity (Patho 1 and Patho 2 in Table 1). These two conditions have been simulated by modifying the model parameters according to qualitative knowledge related to the main mechanisms involved in the pathological changes of breathing mechanics.

Even though each of the millions of alveoli and terminal airways potentially could act as a separate mechanical unit, it is known that, in a pair of normal lungs, the vast majority of these units behave as a single homogeneous entity that includes most lung volume. The few units with different characteristics represent a much smaller compartment, which has little influence on the overall behaviour of the system. Pathological changes can lead to substantial heterogeneity, i.e. abnormal pulmonary systems may require models containing significant combinations of different mechanical units. With reference to the model of Fig. (1), this means that the influence and the weight of a second compartment becomes increasingly greater. 
Table 1. Model Parameter Values

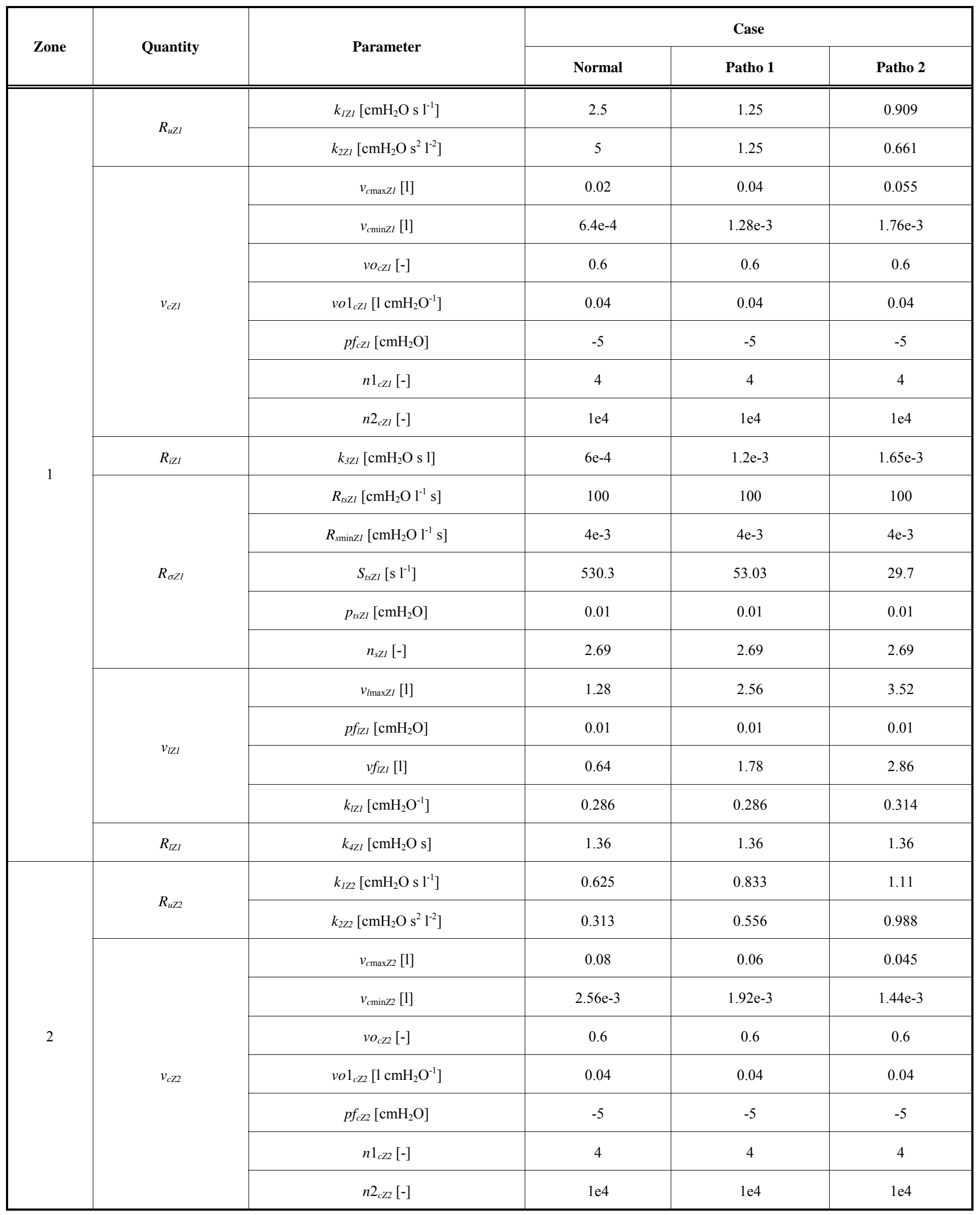


Table 1 Contd.....

\begin{tabular}{|c|c|c|c|c|c|}
\hline Zone & Quantity & Parameter & Normal & Patho 1 & Patho 2 \\
\hline \multirow{7}{*}{2} & \multirow{4}{*}{$R_{\sigma Z 2}$} & $R_{t s Z 2}\left[\mathrm{cmH}_{2} \mathrm{O} \mathrm{l}^{-1} \mathrm{~s}\right]$ & 100 & 100 & 100 \\
\hline & & $S_{t s Z 2}\left[\mathrm{~s} \mathrm{l}^{-1}\right]$ & $1.56 \mathrm{e} 3$ & $1.77 \mathrm{e} 2$ & 74.24 \\
\hline & & $p_{\text {tsZ2 }}\left[\mathrm{cmH}_{2} \mathrm{O}\right]$ & 1.01 & 1.01 & 1.01 \\
\hline & & $n_{s Z 2}[-]$ & 2.69 & 2.69 & 2.69 \\
\hline & \multirow{2}{*}{$v_{I Z 2}$} & $v f_{I Z 2}[1]$ & 0.96 & 0.72 & 0.54 \\
\hline & & $k_{I Z 2}\left[\mathrm{cmH}_{2} \mathrm{O}^{-1}\right]$ & 0.136 & 0.136 & 0.136 \\
\hline & $R_{I Z 2}$ & $k_{4 \mathrm{Z} 2}\left[\mathrm{cmH}_{2} \mathrm{O} \mathrm{s}\right]$ & 1.36 & 1.36 & 1.36 \\
\hline \multicolumn{3}{|c|}{$v_{I \max Z 1}+v_{I \max Z 2} \approx \mathrm{TLC}[1]$} & 6.4 & 6.4 & 6.4 \\
\hline \multicolumn{3}{|c|}{$v f_{I Z 1}+v f_{I Z 2} \approx \mathrm{RV}[1]$} & 1.6 & 2.5 & 3.4 \\
\hline
\end{tabular}

Small airways may suffer early changes, later extending to most bronchial airways. The alterations generally comprise two related diseases, chronic bronchitis and emphysema, one rarely occurring without some degree of the other. These changes lead to an increase in the resistance of involved airways, which is associated with a significant increase in residual volume and functional residual capacity, culminating in a chronically overinflated lung. To mimic such a scenario we accordingly modified the characteristics of the simulation model with respect to the normal case from the trachea to the respiratory zone.

In the normal case (see Table 1), TLC and RV were about 6.4 and $1.6 \mathrm{l}$, respectively (conductive region volumes were neglected with respect to respiratory region volumes [5]). The percentage volumes of $\mathrm{Z} 1$ and $\mathrm{Z2}$ at TLC (i.e. $v_{\text {ImaxZi }}$ TLC $\left.100, i=1,2\right)$ were equal to $20 \%$ and $80 \%$ of TLC, respectively, while the residual volumes of the respiratory region of the two zones $\left(v f_{I Z i} i=1,2\right)$ were set at slightly different values. Finally, the parameters of each zone were defined so as to reproduce a healthy condition. Thus, during quiet breathing, the values chosen for the model parameters led to a resistance value of the conducting airways equal to about $2 \mathrm{cmH}_{2} \mathrm{O} \mathrm{l}^{-1} \mathrm{~s}$ for $\mathrm{Z} 2$. The resistance of $\mathrm{Z} 1$ was about 3 times larger, in agreement with the fact that the number of parallel conducting airways of $\mathrm{Z1}$ is coherently less than that of Z2. The resistance value of the whole model (i.e., Z1+Z2) was equal to approximately $1.5 \mathrm{cmH}_{2} \mathrm{O} \mathrm{l}^{-1} \mathrm{~s}$ and the smaller airway resistance was negligible, corresponding to a typical normal condition.
For the pathological cases, the total lung capacity was set equal to the normal case, but the residual volume was significantly increased to reproduce pathological alterations. In particular, RV was 2.5 and 3.41 for Patho 1 and Patho 2, respectively. Furthermore we increased significantly the weight of $Z 1$, which in the normal case is very small and, therefore, has little influence on the overall behavior of the system. This was done by increasing the volume of the respiratory region of $\mathrm{Z1}$ and simultaneously decreasing that of $\mathrm{Z} 2$ (see Table 1). Thus, the proportion of $Z 1$ grew from $20 \%$ to $40 \%$ of TLC passing from the normal case to Patho 1, and reached 55\% for Patho 2.

Fig. (2) shows the elastic characteristics (in terms of percentage volume) of the respiratory region of lung zones Z1 and Z2 in normal and pathological cases. The percentage volume of each zone (with respect to its maximum admissible volume, i.e. $\left.v_{I Z i} / v_{I \max Z i} \cdot 100\right)$ is plotted against transpulmonary pressure. With reference to Z1, Fig. (2) shows that, for equal pressure, higher percentage volumes are reached by increasing the level of disease; thus, for high levels of disease, the respiratory region of $\mathrm{Z1}$ is more stretched and this results in an overinflated lung. In order to simplify analysis, the elastic characteristics of Z2 was taken to be identical for all cases.

The values chosen for the model parameters corresponded to a remarkable increase in the resistance value of the entire model for Patho 1, and even larger Patho 2, also during quiet breathing. In particular, both Z1 and Z2 showed an increase in the resistances of conducting airways. For ex- 
ample, the resistance of $\mathrm{Z} 2$ was approximately $4 \mathrm{cmH}_{2} \mathrm{O}^{-1} \mathrm{~s}$ and $12 \mathrm{cmH}_{2} \mathrm{O} \mathrm{l}^{-1} \mathrm{~s}$ for Patho 1 and 2, respectively. This considerable increase was largely due to the consequences of significant changes in the smaller airways, whose resistance became no longer negligible passing from the normal case to Patho 1 and 2. From a qualitative point of view, this was fully consistent with pathological changes, which usually have an important impact on smaller airways. Less significant changes interested the upper and intermediate airways. In order to simplify the analysis, the elastic characteristics of the intermediate airways were taken to be equal for the three cases here considered (see Fig. 3).

The whole system was implemented in MATLABSIMULINK using a variable-step solver for stiff problems.

\section{RESULTS AND DISCUSSION}

The results refer to single-breath nitrogen washout reproduced by the proposed functional model with an exhaled air- flow rate $\left(\dot{v}_{0}\right)$ equal to $0.2 \mathrm{l} / \mathrm{s}$ for all cases considered. Fig. (4) shows the fractional concentration of nitrogen in the exhaled volume starting from the beginning of phase III in normal and pathological simulated conditions. The obtained curves point out an almost constant percentage rate of increase in nitrogen concentration for a wide interval of expired volume in phase III of the test.

In the normal case, the slope of the single-breath nitrogen-washout curve remained almost constant for lung volumes from about 6.5 to 2.21 . In this volume range the increase in nitrogen concentration during the test was about $1.1 \%$ per $1000 \mathrm{ml}$ of expired volume. Toward the end of the vital capacity expiration (lung volume of about 2.21 ) there was an abrupt rise in nitrogen concentration, signalling the onset of airway closure or phase IV. The model results show that in this case the onset of phase IV can easily be identified by drawing a straight line through phase III and noting the last point of departure of the nitrogen trace from this line. In particular, the closing volume is equal to about $2.2-$

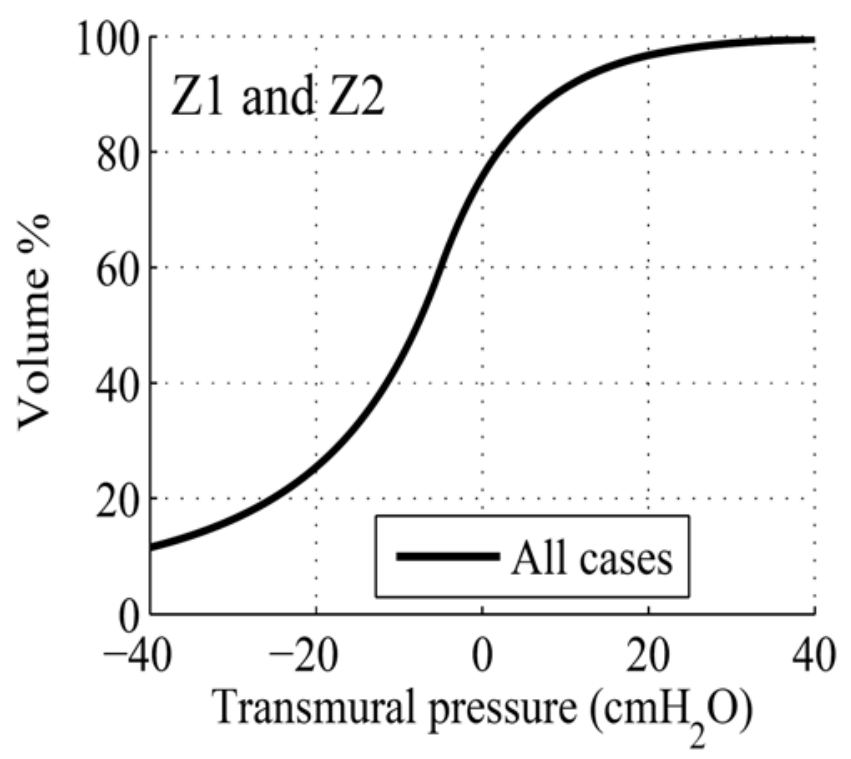

Fig. (3). Elastic characteristics of the intermediate airways. The percentage volume of air in these airways (with respect to their maximum admissible volume) is plotted against transmural pressure.

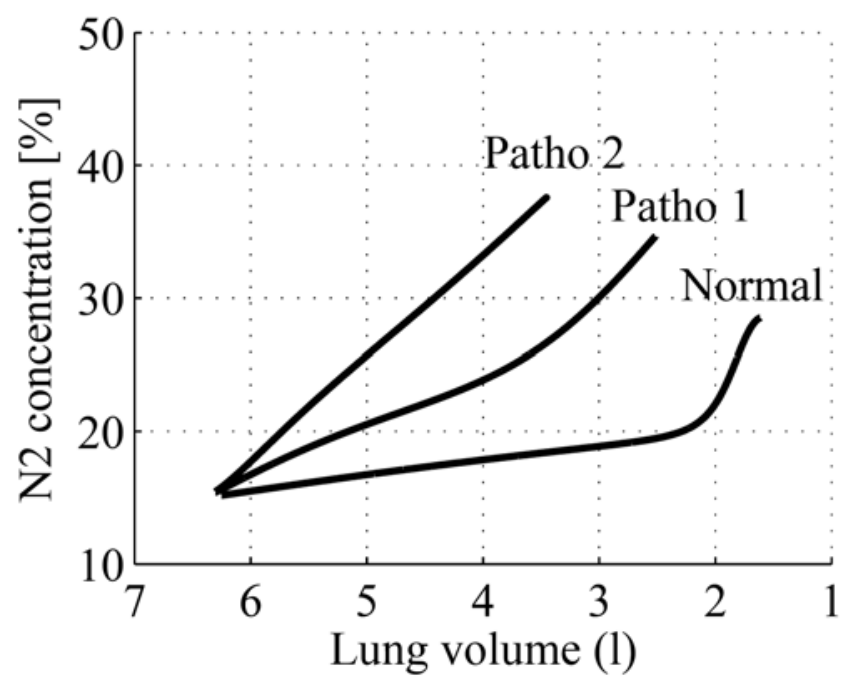

Fig. (4). Fractional concentration of nitrogen in exhaled volume for the three simulated cases starting from the beginning of phase III. 
$1.6=0.61$, which, for this case, corresponds to about $12.5 \%$ of the vital capacity, $\mathrm{VC}(\mathrm{VC}=6.4-1.6=4.81)$. This value of closing volume is close to that of a young healthy man. In fact, in young normal subjects, the closing volume is about $10 \%$ of the vital capacity. It increases steadily with age and is equal to about $40 \%$ of the vital capacity at about the age of 65 years [21].

As in the normal case, the curve of Patho 1 showed two different phases (phase III and IV), but in this case the onset of phase IV could not be easily identified. For lung volumes from about 6.5 to 3.51 the increase in nitrogen concentration was about $3.6 \%$ per $1000 \mathrm{ml}$ of expired volume, indicating a pathological condition.

Finally, the curve of Patho 2 did not show two different phases. In particular, no significant change in the slope of the curve was observed from the beginning of phase III to residual volume (3.4 1 in this case). The rate of increase in nitrogen concentration remained about constant at $7.7 \%$ per 1000 $\mathrm{ml}$ of expired volume, indicating a more severe pathological condition.

Fig. (4) shows that in all cases the slope of the singlebreath nitrogen-washout curve remained almost constant for lung volumes from about 6.5 to $3.5 \mathrm{l}$, but progressively increased with the severity of pathology. This indicates that the nitrogen concentration of the expired alveolar gas was much more uniform in the normal case, reflecting a much more uniform dilution of alveolar gas by the inspired pure oxygen. On the contrary, the slope increased dramatically as pathological condition deteriorated, reflecting uneven dilution of alveolar nitrogen by the inspired pure oxygen. It is well known that the single-breath nitrogen-washout curve is a reliable measure of uneven lung ventilation, which increases in most types of lung diseases [2]. The results obtained by the simulation suggest that the proposed model reproduces phase III of the test under different pathophysiological conditions. Moreover, the slope of phase III did not change appreciably when we used different values of $\dot{v}_{0}$ (less than 0.5 1/s [2]) to simulate a vital capacity expiration.

The reasons for the rise in nitrogen concentration in phase III are not fully understood [2] and the model may help to provide some insights. The values in Table 1 show that the percentage volume changes in $\mathrm{Z} 1$ corresponding to a vital capacity inspiration are $200 \%, 144 \%$ and $123 \%$ for Normal, Patho 1 and Patho 2 cases, respectively. This implies that the fractional concentration of nitrogen in $\mathrm{Z} 1$ at the end of vital capacity inspiration gradually increases between normal and increasingly severe cases. In other words, this zone has a significantly increasing concentration of nitrogen because there is less oxygen to dilute the gas. At the same time, the final volume of $Z 1$ is $20 \%, 40 \%$ and $55 \%$ of total lung capacity for Normal, Patho 1 and Patho 2 cases, respectively. An increasing volume of gas with an increasing nitrogen concentration is therefore exhaled during vital capacity expiration as lung condition deteriorates.

The pattern of outflow from the respiratory region of $\mathrm{Z} 1$ and Z2 during phases III and IV (when present) of vital capacity expiration is shown in Fig. (5) as a function of total lung volume (Please note that the volume is drawn with an inverse axis for consistency with the convention used for the

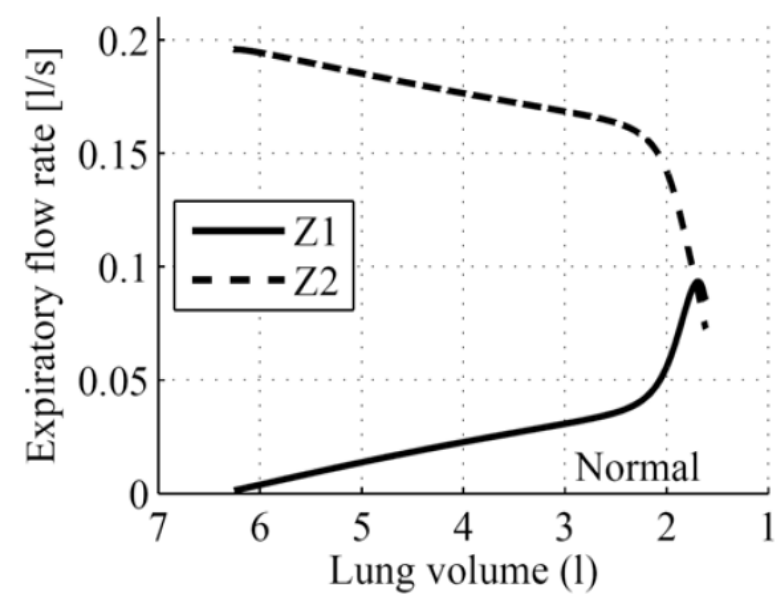

(a)

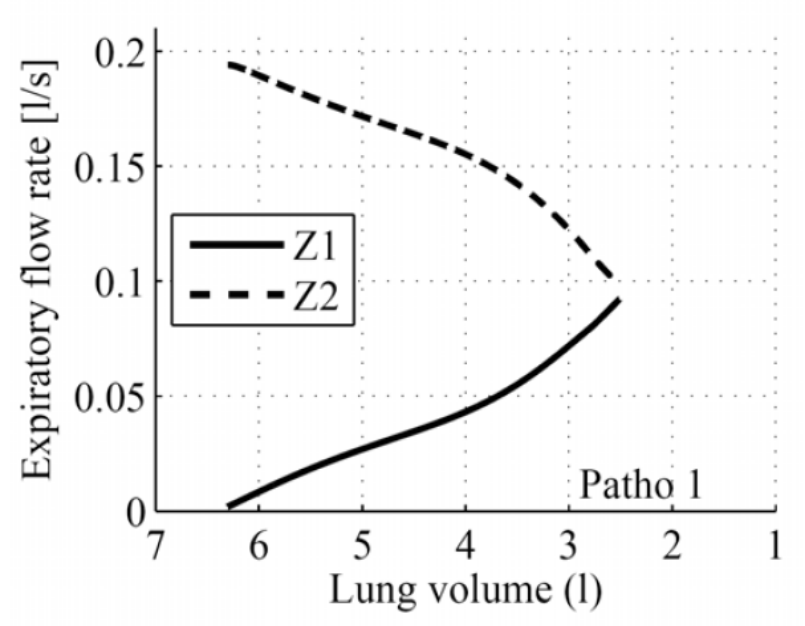

(b)

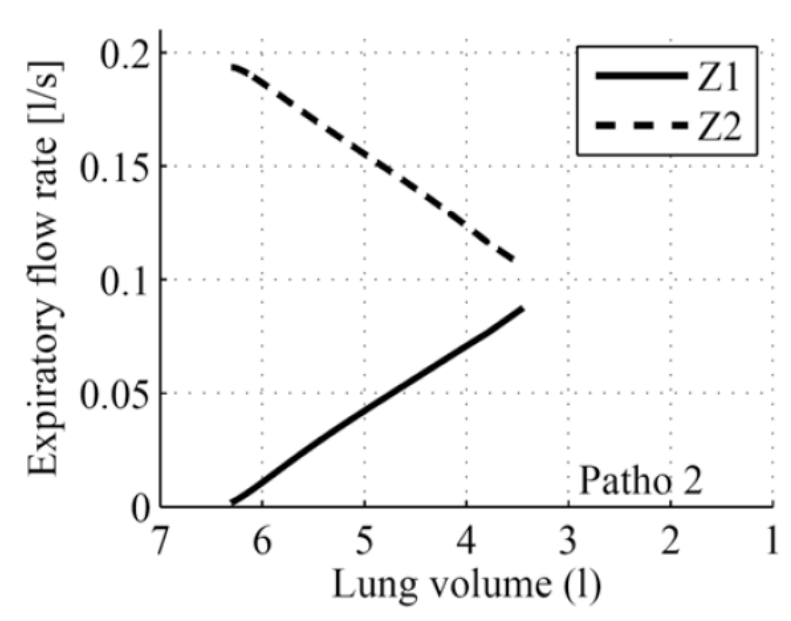

(c)

Fig. (5). Outflow from $\mathrm{Z} 1$ and $\mathrm{Z} 2$ as a function of total lung volume for normal (a) and pathological (b and c) cases. 
single-breath nitrogen washout curves). At the beginning of phase III, expiratory flow came mostly from Z2 in all cases, while outflow from Z1 was close to zero. Outflow from Z1 then rose continually while that from $\mathrm{Z} 2$ decreased, so that their ratio increased significantly, approaching one towards the end of the expiration. This increase was accentuated by severe pathology. As a result, the nitrogen concentration in total exhaled flow and the slope of phase III increased between the normal case and the pathological cases.

Interestingly, in Patho 2 outflow changes from Z1 and Z2 could be represented simply as two straight lines with opposite slopes during the portions of expiration considered. This explains the absence of phase IV in the corresponding singlebreath nitrogen washout test. On the contrary in the normal case the outflow curves from $\mathrm{Z} 1$ and $\mathrm{Z} 2$ showed an abrupt inflection in the final part of expiration. For a given lung volume, flow from Z2 suddenly decreased and that from Z1 increased. As a result, the nitrogen concentration of the expired gas increased abruptly, producing an unambiguous phase IV in the single-breath test. Patho 1 showed an intermediate pattern: the outflow curves from $\mathrm{Z} 1$ and $\mathrm{Z} 2$ did not approximate straight lines; there was a slope change, but the inflection was not so evident.

The slope of phase III significantly changed with increasing obstruction of small airways. The rate of emptying of each zone was determined by its time constant (RC): the larger the time constant, the longer it took to empty. The zone was therefore slow to empty if resistance increased.

Analysis of these results suggests that the proposed model may be useful for investigating the key mechanisms involved in determining different behaviours of the curve observed during the single breath nitrogen test. However, the model has limits due to simplification. A small number of zones is included in the model with respect to real lung structure. Real lungs branch at a number of levels (from the trachea to the alveolar ducts), in contrast to the single branching of the proposed model. Besides, the distributed changes in viscoelastic characteristics of the conducting airways are basically represented using three compartments in series. Finally, the respiratory region is modelled simply as a parallel combination of spring and dashpot. Although a simplified structure is assumed, the number of model parameters is still too large to be determined from the measured data. Selection of the set of parameters explaining the results is therefore to some degree arbitrary and certainly does not exclude other combinations.

Gravity is not explicitly included in the present model, for the sake of simplicity. However, it is remarked that 1) most of the methods for measuring regional ventilation have found that ventilation distribution cannot be accounted for by gravity alone since a large heterogeneity of ventilation within isogravitational planes was observed [22], and 2) the effect of gravity on heterogeneity of respiratory mechanics and gas transport can be seen as incorporated in the different mechanical properties of the lung zones.

\section{CONCLUSION}

The single-breath nitrogen-washout curve is a reliable test for measuring uneven lung ventilation, which increases in most types of lung disease. In the present study a nonlinear dynamic functional model of breathing mechanics was proposed to reproduce the test in different pathophysiological conditions. The simulation obtained with the model showed that phase III slope depends on nitrogen concentration differences between lung zones, as determined by different mechanical properties in the respiratory region of airways.

The normal simulated case showed well-defined phases III and IV. In this condition the slope of the single-breath nitrogen-washout curve remained constant during phase III and the corresponding increase in nitrogen concentration was about $1.1 \%$ per $1000 \mathrm{ml}$ of expired volume. Thus a straight line could be used to approximate changes in the fractional concentration of nitrogen in the exhaled volume during phase III and the last point of departure of the nitrogen trace from this line marked the onset of phase IV.

The slope of phase III progressively increased with the severity of pathology, reflecting much more uneven dilution of alveolar gas by the inspired pure oxygen. The distinction between phases III and IV also became less evident as pathological condition worsened.

These preliminary results indicate that a nonlinear model incorporating two parallel zones, each divided into different series segments, can reproduce a variety of experimental data usually obtained by the single-breath nitrogen washout test in different patients. Simulations performed using the model provided quantitative information about main mechanisms involved in determining the slope of the single-breath nitrogen washout curve during phase III. The fact that the results of these simulations are consistent with the usual results of SBNW test in real situations encourages use of the model to investigate other features that may influence the slope of phase III and the causes determining the presence or absence of phase IV.

In conclusion, although the proposed model is a simplified functional representation of the bronchial tree, it seems to retain key aspects of breathing mechanics which are essential for reproducing and interpreting changes observed during the single-breath nitrogen washout test in normal and pathological patients. The present model appears also useful for teaching and training applications.

\section{GLOSSARY}

$\begin{array}{lll}v_{c Z i} & = & \text { Volume of intermediate airways in zone } \mathrm{Zi} \\ v_{I Z i} & = & \text { Alveolar volume in zone } \mathrm{Zi} \\ v & & \text { Overall lung volume, given by } \\ & v=\sum_{i=1}^{2}\left(v_{l Z i}+v_{c Z i}\right) \\ \mathrm{TLC} & = & \text { Total lung capacity } \\ \mathrm{TLC} & = & \text { Total lung capacity of zone } \mathrm{Zi} \\ \mathrm{RV} & = & \text { Residual volume } \\ \mathrm{RV}_{Z i} & = & \text { Residual volume of zone } \mathrm{Zi} \\ \dot{v} & = & \text { Mouth flow rate }\end{array}$




\begin{tabular}{|c|c|c|}
\hline$\dot{v}_{0}$ & $=$ & $\begin{array}{l}\text { Desired value of expired mouth flow rate } \\
\text { during the single-breath washout test }\end{array}$ \\
\hline$\dot{v}_{Z i}$ & $=$ & Flow rate leaving zone $\mathrm{Zi}$ \\
\hline$\dot{v}_{l Z i}$ & $=$ & $\begin{array}{l}\text { Flow rate leaving the respiratory region of } \\
\text { zone } \mathrm{Zi}\end{array}$ \\
\hline$\dot{V}_{l}$ & $=$ & $\begin{array}{l}\text { Total airflow rate from the respiratory re- } \\
\text { gion }\left(=\dot{v}_{l Z 1}+\dot{v}_{I Z 2}\right)\end{array}$ \\
\hline$p^{t m} m_{Z i}$ & $=$ & $\begin{array}{l}\text { Transmural pressure in the intermediate } \\
\text { airways of zone } \mathrm{Zi}\end{array}$ \\
\hline$p_{E l Z i}$ & $=$ & $\begin{array}{l}\text { Difference between alveolar pressure and } \\
\text { pleural pressure in zone } \mathrm{Zi}\end{array}$ \\
\hline$P_{p l}$ & $=$ & Pleural pressure \\
\hline$R_{u Z i}$ & $=$ & Resistance of upper airways in zone $\mathrm{Zi}$ \\
\hline$R_{i Z i}$ & $=$ & $\begin{array}{l}\text { Resistance of intermediate airways in zone } \\
\mathrm{Zi}\end{array}$ \\
\hline$R_{s Z i}$ & $=$ & Resistance of smaller airways in zone $\mathrm{Zi}$ \\
\hline
\end{tabular}

$\begin{array}{ll}R_{l Z i} & =\begin{array}{l}\text { Resistance of the respiratory region in } \\ \text { zone } \mathrm{Zi}\end{array} \\ E_{a w Z i} & =\begin{array}{l}\text { Elastance of intermediate airways in zone } \\ \mathrm{Zi}\end{array} \\ E_{I Z i} & =\quad \begin{array}{l}\text { Elastance of the alveolar space in zone } \mathrm{Zi} \\ F_{\mathrm{N} 2}\end{array} \\ F_{\mathrm{N} 2 l Z i}= & \begin{array}{l}\text { Fractional concentration of nitrogen in ex- } \\ \text { pired mixed gas }\end{array} \\ & \begin{array}{l}\text { Nitrogen fractional concentration in the } \\ \text { respiratory region of zone } \mathrm{Zi}\end{array}\end{array}$

\section{CONFLICT OF INTEREST}

The authors confirm that this article content has no conflicts of interest.

\section{ACKNOWLEDGMENTS}

This work was partly financed by the Italian Ministry of Education, University and Research.

\section{APPENDIX}

\section{A.1. Tracheobronchial Tree Model}

Upper airways are described using a nonlinear Rohrer resistor:

$R_{u Z i}=k_{1 Z i}+k_{2 Z i}\left|\dot{v}_{Z i}\right|$

where constants $k_{1 Z i}$ and $k_{2 Z i}$ are parameters empirically describing the pressure-flow relationship [18].

The intermediate airways are represented as a collapsible segment of constant length [13], the volume $\left(v_{c Z i}\right)$ of which varies with transmural pressure $\left(\mathrm{ptm}_{\mathrm{Zi}}\right)$ according to a sigmoidal elastic curve based on that proposed by Lambert [19]:

$$
\begin{aligned}
& v_{c Z i}=\left(v_{c \max Z i} \cdot v o_{c Z i}-v_{c \min Z i}\right) \cdot\left(1-\frac{v o 1_{c Z i} \cdot v_{c \max Z i} \cdot\left(p t m_{Z i}-p f_{c Z i}\right)}{n 1_{c Z i} \cdot\left(v_{c \max Z i} \cdot v o_{c Z i}-v_{c \min Z i}\right)}\right)^{-n 1_{c Z i}}+v_{c \min Z i} \quad \text { for } p t m_{Z i}<p f_{c Z i} \\
& v_{c Z i}=v_{c \max Z i} \cdot\left(1-\left(1-v o_{c Z i}\right) \cdot\left(1-v 1_{c Z i} \cdot \frac{p t m_{Z i}-p f_{c Z i}}{n 2_{c Z i} \cdot\left(v o_{c Z i}-1\right)}\right)^{-n 2_{c Z i}}\right) \text { for } p t m_{Z i} \geq p f_{c Z i},
\end{aligned}
$$

where $v O_{c Z i}=\left.\left(v_{c Z i} / v_{c \max Z i}\right)\right|_{p t m_{Z i}=p f_{c Z i}}$ and $v o 1_{c Z i}=\left.\left(\mathrm{d} v_{c Z i} / \mathrm{d} v_{c \max Z i}\right)\right|_{p t m_{Z i}=p f_{c Z i}}$, while $v_{c \operatorname{maxZi}}$ and $v_{c \min Z i}$ are maximum and minimum admissible volumes of air in these airways, $p f_{c Z i}$ is the value of $p t m_{Z i}$ at the inflexion point and $n 1_{c Z i}$ and $n 2_{c Z i}$ are constants. (Note: Although intermediate airway volume is negligible with respect to overall lung volume during a vital-capacity maneuver (only a few percent [5]), it is considered to evaluate the elastic and viscous properties of this airway segment).

Smaller airways are represented by a nonlinear resistance $\left(R_{S Z i}\right)$ which increases when transpulmonary pressure $\left(p_{E I Z i}\right)$ decreases. When $p_{E I Z i}$ falls below the threshold value $\left(p_{\mathrm{tsZ} i}\right)$, they collapse; i.e. their resistance goes to infinity and the airflow out of the alveoli $\left(\dot{v}_{I Z i}\right)$ is zero. Residual volume was assumed to be the volume of air in the lungs when the smaller airways are completely closed. For $p_{E I Z i} \geq p_{\mathrm{tsZi}}$ smaller airways resistance is given by:

$$
R_{\mathrm{s} Z i}=\left(R_{\mathrm{ts} Z i}-R_{\mathrm{s} \min Z i}\right) \cdot\left(1-\frac{S_{\mathrm{ts} Z i} \cdot\left(p_{\mathrm{ts} Z i}-p_{E l Z i}\right)}{n_{\mathrm{s} Z i} \cdot\left(R_{\mathrm{ts} Z i}-R_{\mathrm{s} \min Z i}\right)}\right)^{-n_{\mathrm{s} Z i}}+R_{\mathrm{s} \min Z i}
$$

where $R_{\mathrm{tsZi}}$ and $S_{\mathrm{tsZi}}$ are smaller airways resistance and its slope when transpulmonary pressure is equal to its threshold value, respectively, while $R_{\text {sminzi }}$ is the minimum value of smaller airways resistance and $n_{s Z i}$ is a constant. In simulating a vitalcapacity inspiration, we assumed that the smaller airways resistance again becomes finite and is described by equation (A3) when pleural pressure equals $-p_{\mathrm{ts} z i}$.

The elastance of the alveolar space $\left(E_{I Z i}\right)$ is obtained by differentiating $p_{E I Z i}$ with respect to $v_{I Z i}$, where the relationship between $v_{I Z i}$ and $p_{E I Z i}$ is represented by an exponential curve based on the Glaister equation [20]:

$v_{l Z i}=v_{l \max Z i}-\left(v_{l \max Z i}-v f_{I Z i}\right) \cdot e^{-k_{I Z i} \cdot\left(p_{E I Z i}-p f_{I Z i}\right)}$ 
where $v_{I \max Z i}$ is the maximum admissible volume of air in the alveolar space of the $i$-th zone, $p f_{I Z i}$ and $v f_{I Z i}$ are the values of $p_{E I Z i}$ and $v_{I Z i}$ when the smaller airways completely close and $k_{I Z i}$ is a constant proportional to the slope of the curve at $p f_{I Z i}$.

\section{A.2. Intrapleural Pressure Time Course}

\section{A.2.1. Tidal Respiration}

The time course of intrapleural pressure during a tidal breath is divided into three parts. At the beginning of inspiration $(t=$ 0 and lung volume $=$ functional residual capacity, FRC), pleural pressure equals its rest value, $P p l_{F R C}$. During inspiration it decreases and reaches a minimum at $t=t_{P p l \min }$. During expiration, it rises, reaching $P p l_{F R C}$ after a set time interval $\Delta t$, whereupon it remains constant until the next inspiration. In detail

$P p l=P p l_{F R C}+\frac{\Delta P p l_{F R C}}{t_{P p l \min }^{2}} \cdot t^{2}-2 \cdot \frac{\Delta P p l_{F R C}}{t_{P p l \min }} \cdot t$

for $0 \leq t \leq t_{P p l \text { min }}$

$P p l=P p l_{F R C}-\frac{\Delta P p l_{F R C}}{\Delta t^{2}} \cdot t^{2}+2 \cdot \frac{\Delta P p l_{F R C} \cdot\left(t_{P p l \text { min }}+\Delta t\right)}{\Delta t^{2}} \cdot t-\frac{\Delta P p l_{F R C} \cdot\left(t_{P p l \text { min }}+\Delta t\right)^{2}}{\Delta t^{2}}$

for $t_{P p l \min } \leq t \leq t_{P p l \min }+\Delta t$,

$P p l=P p l_{F R C}$

for $t_{P p l \min }+\Delta t \leq t \leq T$

where $T$ is the respiratory period and $\triangle P p l_{F R C}$ is the total variation in intrapleural pressure during the time interval from 0 to $t_{P p l \text { min. }}$. During each simulation, 20 respiratory cycles were reproduced to reach a steady-state periodic condition [23].

\section{A.2.2. Expiration to Residual Volume.}

After a tidal inspiration, described by equation (A5), an expiration was simulated to residual volume, using the following relationship for intrapleural pressure:

$P p l=P p l_{F R C}+\frac{\Delta P p l_{R V}}{2} \cdot \sin \left(\frac{\pi}{\Delta t_{R V}} t+\frac{3 \pi}{2}-\frac{\pi}{\Delta t_{R V}} \cdot t_{P p l \min }\right)+\frac{\Delta P p l_{R V}}{2}-\Delta P p l_{F R C}$

for $t_{P p l \min } \leq t \leq t_{P p l \min }+\Delta t_{R V}$

where $\Delta P p l_{R V}$ is the total variation in intrapleural pressure from $t_{P p l \min }$ to $t_{P p l \min }+\Delta t_{R V}$. Intrapleural pressure reaches its maximum $\left(P p l_{F R C}+\triangle P p l_{R V}-\triangle P p l_{F R C}\right)$ at the end of maximum expiration which occurs at $t_{P p l \min }+\Delta t_{R V}$.

\section{A.2.3. Single-breath Nitrogen Washout Test}

The single-breath nitrogen washout maneuver consists of two parts. After reaching a steady state breathing air, the subject inspires $100 \%$ oxygen from residual volume to total lung capacity (vital-capacity inspiration). After a momentary pause at TLC, the individual exhales very slowly to RV (slow vital-capacity expiration). During the vital-capacity inspiration from RV $(t=0)$ to TLC $\left(t=t_{T L C}\right)$ the time course of intrapleural pressure is modelled as

$P p l=P p l_{F R C}-\frac{\Delta P p l_{T L C}}{t_{T L C}} \cdot t \cdot \exp \left(1-t / t_{T L C}\right)+\Delta P p l_{R V}-\Delta P p l_{F R C}$

which implies a total variation in $P p l$ equal to $\triangle P p l_{T L C}$.

During the slow vital-capacity expiration a feedback integral controller was used to obtain a nearly constant expiratory airflow. Thus in this time interval intrapleural pressure behaves as a further state variable and the system is described by a further differential equation

$\frac{\mathrm{d} P p l}{\mathrm{~d} t}=-k_{\mathrm{C}}\left(\dot{v}-\dot{v}_{0}\right)$

where $\dot{v}$ and $\dot{v}_{0}$ are the actual and desired constant expiratory airflow rates, respectively, while $k_{\mathrm{C}}$ is a constant large enough to reach $\dot{v}_{0}$ in a sufficiently short time. The initial value of this additional state variable Ppl was taken as being equal to intrapleural pressure at $t=t_{T L C}$, while $k_{\mathrm{C}}$ was set at $10^{4} \mathrm{cmH}_{2} \mathrm{O}^{-1}$.

\section{REFERENCES}

[1] J.H. Comroe Jr, and W.S. Fowler, "Lung function studies. VI. Detection of uneven alveolar ventilation during a single breath of oxygen", Am. J. Med., vol. 10, pp. 408-413, April 1951.
[2] J.B. West, Pulmonary Pathophysiology - The Essentials, Lippincott Williams \& Wilkins, Baltimore 2008.

[3] M. Paiva, and L.A. Engel, "Theoretical studies of gas mixing and ventilation distribution in the lung", Physiol. Rev., vol. 67, pp. 750796, July 1987. 
[4] J. Pardaens, K.P. van de Woestijne, and J. Clément, "Simulation of regional lung emptying during slow and forced expirations", $J$. Appl. Physiol., vol. 39, pp. 191-198, August 1975.

[5] E.R. Weibel, Morphometry of the Human Lung, Springer-Verlag, Berlin 1963.

[6] S.C.M. Luijendijk, A. Zwart, W.R. de Vries, and W.M. Salet, "The sloping alveolar plateau at synchronous ventilation", Pflugers Arch., vol.384, pp. 267-277, April 1980.

[7] M. Paiva, and L.A. Engel, "The anatomical basis for the sloping $\mathrm{N}_{2}$ plateau”, Respir. Physiol., vol. 44, pp. 325-337, June 1981.

[8] M. Paiva, and L.A. Engel, "Model analysis of gas distribution within human lung acinus", J. Appl. Physiol., vol. 56, pp. 418-425, February 1984.

[9] B. Dutrieue, F. Vanholsbeeck, S. Verbanck, and M. Paiva, "A human acinar structure for simulation of realistic alveolar plateau slopes", J. Appl. Physiol., vol.89, pp. 1859-1867, November 2000.

[10] K. Kaneko, "Simultaneous helium and nitrogen single-breath washout: lung model simulation", J. Appl. Physiol., vol. 44, pp. 499-506, April 1978.

[11] M.H. Tawhai, and P.J. Hunter, "Multibreath washout analysis: modelling the influence of conducting airway asymmetry", Respir. Physiol., vol. 127, pp. 249-258, September 2001.

[12] R. Arieli, F. Wiener, and E. Levitan, "Cardiogenic oscillation and phase III caused by pressure-volume heterogeneity: a model", J. Appl. Physiol., vol. 60, pp. 2101-2108, June 1986.

[13] P. Barbini, G. Cevenini, and G. Avanzolini, "Nonlinear mechanisms determining expiratory flow limitation in mechanical ventilation: a model-based interpretation", Ann. Biomed. Eng., vol. 31, pp. 908-916, September 2003.

[14] C. Hook, M. Meyer, and J. Piiper, "Model simulation of singlebreath washout of insoluble gases from dog lungs", J. Appl. Physiol., vol. 58, pp. 802-811, March 1985.
[15] H. Kitaoka, and I. Kawase, "A novel interpretation of closing volume based on single-breath nitrogen washout curve simulation", $J$. Physiol. Sci., vol. 57, pp. 367-376, December 2007.

[16] C. H. Liu, S.C. Niranjan, J.W. Clark Jr, K.Y. San, J.B. Zwischenberger, and A. Bidani, "Airway mechanics, gas exchange, and blood flow in a nonlinear model of the normal human lung", $J$. Appl. Physiol., vol. 84, pp.1447-1469, April 1998.

[17] Y.C. Zhao, S.E. Rees, S. Kjaergaard, and S. Andreassen, "Simulation of pulmonary pathophysiology during spontaneous breathing", in Conf. Proc. IEEE Eng. Med. Biol. Soc., vol. 6, 2005, pp. 61286131.

[18] F. Rohrer, " Der Strömungswiderstand in den menschlichen Atemwegen und der Einfluss der unregelmässigen Verzweigung des Bronchialsystems auf den Atmungsverlauf in verschiedenen Lungenbezirken ", Arch. Ges. Physiol., vol. 162, pp. 225-299, October 1915.

[19] R.K. Lambert, T.A. Wilson, R.E. Hyatt, and J.R. Rodarte, "A computational model for expiratory flow”, J. Appl. Physiol., vol. 52, pp. 44-56, January 1982.

[20] D.H. Glaister, R.C. Schroter, M.F. Sudlow, and J. Milic-Emili, "Bulk elastic properties of excised lungs and the effect of a transpulmonary pressure gradient", Respir Physiol, vol. 17, pp. 347364, April 1973.

[21] J.B. West, Respiratory Physiology - The Essentials. Lippincott Williams \& Wilkins, Baltimore 2012.

[22] K.L. Steimle, M.L. Mogensen, D.S. Karbing, J. Bernardino de la Serna, and S. Andreassen, "A model of ventilation of the healthy human lung", Comput. Methods Prog Biomed., vol. 101, pp. 144155, February 2011.

[23] G. Avanzolini, P. Barbini, F. Bernardi, G. Cevenini, and G. Gnudi, "Role of the mechanical properties of tracheobronchial airways in determining the respiratory resistance time course", Ann. Biomed. Eng., vol. 29, pp. 575-586, July 2001.

Received: November 22, 2012

Revised: February 09, 2013

Accepted: February 11, 2012

(C) Barbini et al.; Licensee Bentham Open.

This is an open access article licensed under the terms of the Creative Commons Attribution Non-Commercial License (http://creativecommons.org/licenses/by-nc/3.0/) which permits unrestricted, non-commercial use, distribution and reproduction in any medium, provided the work is properly cited. 